\title{
Nikodým-Type Theorems for Lattice Group-Valued Measures with Respect to Filter Convergence
}

\author{
Antonio Boccuto ${ }^{1}$, Xenofon Dimitriou ${ }^{2 *}$ \\ ${ }^{1}$ Dipartimento di Matematica e Informatica, Perugia, Italy \\ ${ }^{2}$ Department of Mathematics, University of Athens, Athens, Greece \\ Email: xenofon11@gmail.com, dxenof@windtools.gr, ${ }^{*}$ dxenof@math.uoa.gr \\ Received 18 March 2014; revised 18 April 2014; accepted 26 April 2014 \\ Copyright (C) 2014 by authors and Scientific Research Publishing Inc. \\ This work is licensed under the Creative Commons Attribution International License (CC BY). \\ http://creativecommons.org/licenses/by/4.0/ \\ (c) () Open Access
}

\begin{abstract}
We present some convergence and boundedness theorems with respect to filter convergence for lattice group-valued measures. We give a direct proof, based on the sliding hump argument. Furthermore we pose some open problems.
\end{abstract}

\section{Keywords}

Lattice Group, (Free) Filter, ( $s$ )-Bounded Measure, $\sigma$-Additive Measure, Diagonal Filter, Block-Respecting Filter, Limit Theorem, Nikodým Boundedness Theorem

\section{Introduction}

In the literature there have been several recent studies about limit theorems with respect to filter/ideal convergence for measures, taking values in abstract spaces, whose a particular case is the statistical convergence, related with asymptotic density of subsets of the set of natural numbers. Though in general it is not possible to give versions of limit theorems completely analogous to the corresponding classical ones in the filter/ideal setting (see also [1], Example 3.4), there are different kinds of results on these topics, whose an overview can be found in [2] and its bibliography, together with a historical survey on several types of kinds of such theorems and related topics since the beginning of the last century. Some classical like limit theorems for measures and integrals in the context of lattice groups or similar structures can be found, for instance, in [3]-[7]. In particular, in [1] [8], some versions of basic matrix theorems are given, extending results of [9] [10], which were proved in the normed space context and with respect to statistical convergence. In [11]-[17], some Schur, boundedness,

*Corresponding author. 
decomposition and/or convergence theorems are given in the lattice group setting with respect to a suitable class of filters, extending some results of [18], while for positive measures it is possible to have some similar results even for a larger class of filters (see also [19]). Analogous results have been established also in the setting of topological group-valued measures in [20] [21]. In [22]-[24], some limit theorems are proved by means of the tool of uniform filter/ideal exhaustiveness. Moreover, in [25], some results about equivalence between BrooksJewett, Vitali-Hahn-Saks, Nikodým convergence and Dieudonné-type theorems are presented, using the Stone Isomorphism technique and extending results of [26], given in the classical case for topological group-valued measures. In this paper we use sliding hump-type techniques, similar to those used in the topological group-setting first by D. Candeloro and G. Letta in 1985 in [27]-[30] for proving limit and boundedness theorems for families of finitely additive group-valued measures defined on suitable Boolean algebras, and successively in [4] [5] [16] [17] [20] [21] in the setting of lattice groups and filter convergence. We prove some new further versions of Nikodým convergence, boundedness and Brooks-Jewett-type theorems for lattice group-valued measures, defined on a $\sigma$-algebra of an abstract nonempty set. The results and the proofs are direct and, differently than in [14] [16], without using Schur-type theorems proved for measures defined on $\mathcal{P}(\mathbb{N})$. Finally, we pose some open problems.

\section{Preliminaries}

A lattice group (shortly, $(\ell)$-group) $R$ is said to be Dedekind complete iff every nonempty subset of $R$, bounded from above, admits supremum in $R$. A Dedekind complete lattice group is said to be super Dedekind complete iff for every nonempty set $A \subset R$, bounded from above, there exists a countable subset $A^{*} \subset A$, such that $\vee A=\vee A^{*}$.

Let $R$ be a Dedekind complete $(\ell)$-group. A sequence $\left(\sigma_{p}\right)_{p}$ of positive elements of $R$ is called an $(O)$-sequence iff it is decreasing and $\wedge_{p} \sigma_{p}=0$. A sequence $\left(x_{n}\right)_{n}$ in $R$ is said to be order convergent (or $(O)$-convergent) to $x \in R$ iff there exists an $(O)$-sequence $\left(\sigma_{p}\right)_{p}$ in $R$ such that for every $p \in \mathbb{N}$ there is a positive integer $n_{0}$ with $\left|x_{n}-x\right| \leq \sigma_{p}$ for all $n \geq n_{0}$, and in this case we will write $(O) \lim _{n} x_{n}=x$. A bounded double sequence $\left(a_{t, l}\right)_{t, l}^{n}$ in $R$ is called a $(D)$-sequence or a regulator iff for each $t \in \mathbb{N}$ the sequence $\left(a_{t, l}\right)_{1}$ is an $(O)$-sequence. A sequence $\left(x_{n}\right)_{n}$ in $R$ is said to be $(D)$-convergent to $x \in R$ (and we write $\left.(D) \lim _{n} x_{n}=x\right)$ iff there exists a $(D)$-sequence $\left(a_{t, l}\right)_{t, l}$ in $R$, such that to every $\varphi \in \mathbb{N}^{\mathbb{N}}$ there is $n_{0} \in \mathbb{N}$ with $\left|x_{n}-x\right| \leq \vee_{t=1}^{\infty} a_{t, \varphi(t)}$ for every $n \geq n_{0}$. An $(\ell)$-group $R$ is weakly $\sigma$-distributive iff $\wedge_{\varphi \in \mathbb{N}^{\mathbb{N}}}\left(\vee_{t=1}^{\infty} a_{t, \varphi(t)}\right)=0$ for every $(D)$-sequence $\left(a_{t, l}\right)_{t, l}$.

Observe that, if $\Sigma$ is the $\sigma$-algebra of all Borel subsets of $[0,1]$ and $v$ is the Lebesgue measure, then the space $L^{0}([0,1], \Sigma, v)$ of all $v$-measurable real-valued functions on $[0,1]$ (with identification up to $v$ -null sets) is super Dedekind complete and weakly $\sigma$-distributive (see also [5], [6], Example 2.17).

We now recall the following theorem, which links $(O)$ - and $(D)$-sequences in lattice groups.

Theorem 2.1 ([25], Theorem 2.3, see also [31], Theorems 3.1 and 3.4) Given any Dedekind complete $(\ell)$ -group $R$ and any $(O)$-sequence $\left(\sigma_{l}\right)_{l}$ in $R$, the double sequence defined by $a_{t, l}:=\sigma_{l}, t, l \in \mathbb{N}$, is $a$ regulator, such that for every $\varphi \in \mathbb{N}^{\mathbb{N}}$, if $l=\varphi(1)$, then $\sigma_{l} \leq \underset{t=1}{\vee_{t, \varphi(t)}}$. Conversely, if $R$ is super Dedekind complete and weakly $\sigma$-distributive, then for every $(D)$-sequence $\left(a_{t, l}\right)_{t, l}$ in $R$ there are an $(O)$-sequence $\left(b_{k}\right)_{k}$ in $R$ and a sequence $\left(\varphi_{k}\right)_{k}$ in $\mathbb{N}^{\mathbb{N}}$, with $\underset{t=1}{\vee} a_{t, \varphi_{k}(t)} \leq b_{k}$ for each $k$.

Let $G$ be any nonempty set and $\Sigma \subset \mathcal{P}(G)$ be a $\sigma$-algebra. A bounded finitely additive measure $m: \Sigma \rightarrow \mathbb{R}$ is said to be $\sigma$-additive (resp. (s)-bounded) on $\Sigma$ iff $(D) \lim _{n} v(m)\left(\bigcup_{l=n}^{\infty} H_{l}\right)=0$ (resp. $(D) \lim _{n} v(m)\left(H_{n}\right)=0$ ) for each disjoint sequence $\left(H_{n}\right)_{n}$ in $\Sigma$, where $v(m)(E):=\vee\{|m(A)|: A \in \Sigma, A \subset E\}$, $E \in \Sigma$, is the semivariation of $m$ on $\Sigma$. Moreover, if $G$ is a compact Hausdorff topological space and $\Sigma$ is its Borel $\sigma$-algebra, we say that a positive finitely additive measure $m: \Sigma \rightarrow R$ is regular iff for every 
$E \in \Sigma$ there exists a $(D)$-sequence $\left(a_{i, j}\right)_{i, j}$, depending on $E$, such that for all $\varphi \in \mathbb{N}^{\mathbb{N}}$ there are a compact set $K$ and an open set $U$ with $K \subset E \subset U$ and $m(U \backslash K) \leq \vee_{i=1}^{\infty} a_{i, \varphi(i)}$. We recall the following.

Theorem 2.2 ([32], Theorem 2.2) Assume that $R$ is a Dedekind complete and weakly $\sigma$-distributive Riesz space, and let $m: \Sigma \rightarrow R$ be a regular measure defined on the Borel $\sigma$-algebra of a compact Hausdorff space $G$. Then $m$ is $\sigma$-additive.

The following result (Fremlin lemma, see [33], Lemma 1C) allows us to replace a countable family or a "series" of $(D)$-sequences with a single regulator.

Lemma 2.3 Let $R$ be any Dedekind complete $(\ell)$-group and $\left(a_{t, l}^{(n)}\right)_{t, l}, \quad n \in \mathbb{N}$, be a sequence of regulators in $R$. Then for every $u \in R, u \geq 0$ there exists a $(D)$-sequence $\left(a_{t, l}\right)_{t, l}$ in $R$ with $u \wedge\left(\sum_{n=1}^{q}\left(\bigvee_{t=1}^{\infty} a_{t, \varphi(t+n)}^{(n)}\right)\right) \leq \bigvee_{t=1}^{\infty} a_{t, \varphi(t)}$ for every $q \in \mathbb{N}$ and $\varphi \in \mathbb{N}^{\mathbb{N}}$.

We now give some basic properties of filters, which will be useful in the sequel. Let $Q$ be a countable set and $\mathcal{F}$ be a filter of $Q$. A subset of $Q$ is $\mathcal{F}$-stationary iff it has nonempty intersection with every element of $\mathcal{F}$. We denote by $\mathcal{F}^{*}$ the family of all $\mathcal{F}$-stationary subsets of $Q$.

A filter $\mathcal{F}$ of $Q$ is said to be diagonal iff for every sequence $\left(A_{n}\right)_{n}$ in $\mathcal{F}$ and for each $I \in \mathcal{F}^{*}$ there exists a set $J \subset I, \quad J \in \mathcal{F}^{*}$ such that the set $J \backslash A_{n}$ is finite for all $n \in \mathbb{N}$ (see also [15] [16] [18]). Given an infinite set $I \subset Q$, a blocking of $I$ is a countable partition $\left\{D_{k}: k \in \mathbb{N}\right\}$ of $I$ into nonempty finite subsets. A filter $\mathcal{F}$ of $Q$ is said to be block-respecting iff for every $I \in \mathcal{F}^{*}$ and for each blocking $\left\{D_{k}: k \in \mathbb{N}\right\}$ of $I$ there is a set $J \in \mathcal{F}^{*}, J \subset I$ with $\#\left(J \cap D_{k}\right)=1$ for all $k \in \mathbb{N}$, where \# denotes the number of elements of the set into brackets. A particular class of filters, which are block-respecting and diagonal at the same time, is that of the category respecting filters. A filter $\mathcal{F}$ of $\mathbb{N}$ is said to be category respecting iff for every compact metric space $K$ and for every family of closed subsets $\left(F_{A}\right)_{A \in \mathcal{F}}$ of $K$, if $F_{A} \subset F_{B}$ whenever $B \subset A$ in $\mathcal{F}$ and $K=\bigcup_{A \in \mathcal{F}} F_{A}$, then there is a set $B \in \mathcal{F}$ such that the interior of $F_{B}$ is nonempty (see also [18], Theorem 4.3).

Let $D=\left(D_{n}\right)_{n}$ be a disjoint partition of $\mathbb{N}$ into infinite subsets. For each sequence $C=\left(C_{n}\right)_{n}$ of finite subsets $C_{n} \subset D_{n}$ and every $q \in \mathbb{N}$, set $B_{q, C}:=\bigcup_{n=q}^{\infty}\left(D_{n} \backslash C_{n}\right)$ The filter $\mathcal{F}_{D}$ generated by the sets of type $B_{q, C}$ is a non-diagonal and block-respecting filter. Furthermore, note that the filter of all subsets of $\mathbb{N}$ having asymptotic density one is a diagonal and not block-respecting filter (see also [18]).

If $I \in \mathcal{F}^{*}$, then the trace $\mathcal{F}(I)$ of $\mathcal{F}$ on $I$ is the family $\{A \cap I: A \in \mathcal{F}\}$. It is not difficult to see that $\mathcal{F}(I)$ is a filter of $I$ (see also [21]).

Remark 2.4 Observe that, if $\mathcal{F}$ is a block-respecting filter of $\mathbb{N}$, then $\mathcal{F}(I)$ is a block-respecting filter of $I$ for every $I \in \mathcal{F}^{*}$ (see also [20], Proposition 2.1, [21], Proposition 2.3).

We now recall some main properties of filter convergence in the lattice group setting (see also [1] [16]).

Let $\mathcal{F}$ be a filter of $\mathbb{N}$. A sequence $\left(x_{n}\right)_{n}$ in $R(D \mathcal{F})$-converges to $x \in R$ iff there is a $(D)$-sequence $\left(a_{t, l}\right)_{t, l}$ with the property that $\left\{n \in \mathbb{N}:\left|x_{n}-x\right| \leq \vee_{t=1}^{\infty} a_{t, \varphi(t)}\right\} \in \mathcal{F}$ for each $\varphi \in \mathbb{N}^{\mathbb{N}}$.

Let $\Xi$ be any arbitrary nonempty set. A family $\left(\beta_{\xi, n}\right)_{\xi \in \Xi, n \in \mathbb{N}}$ is said to be $(R D \mathcal{F})$-convergent to a family $\left(\beta_{\xi}\right)_{\xi \in \Xi}$ with respect to $\xi \in \Xi$ iff there is a regulator $\left(a_{t, l}\right)_{t, l}$ such that for each $\varphi \in \mathbb{N}^{\mathbb{N}}$ and $\xi \in \Xi$ we get $\left\{n \in \mathbb{N}:\left|\beta_{\xi, n}-\beta_{\xi}\right| \leq \vee_{t=1}^{\infty} a_{t, \varphi(t)}\right\} \in \mathcal{F}$.

Given $a<b \in R$, set $[a, b]=\{x \in R: a \leq x \leq b\}$. For $A, B \subset R, n \in \mathbb{N}$, put $A+B=\{a+b: a \in A, b \in B\}$, $n A=\{a+\cdots+a\}$ ( $n$ times). Let $U_{n}=\left[-u_{n}, u_{n}\right], n \in \mathbb{N}$, be such that $0<u_{n} \leq u_{n+1}$ for every $n \in \mathbb{N}$. A set $\left\{x_{n}: n \in \mathbb{N}\right\} \subset R$ is said to be $(P R)-\mathcal{F}$-bounded by $\left(U_{n}\right)_{n}$, iff $\left\{n \in \mathbb{N}: x_{n} \in U_{n}\right\} \in \mathcal{F}$, and $(P R)$-eventually bounded by $\left(U_{n}\right)_{n}$ iff it is $(P R)-\mathcal{F}_{\text {cofin }}$-bounded by $\left(U_{n}\right)_{n}^{n}$ (see also [1] [15] [16] [34]).

\section{The Main Results}

We begin with recalling the following

Lemma 3.1 ([16], Lemma 2.3) Let $\mathcal{F}$ be a diagonal filter of $\mathbb{N},\left(a_{i}\right)_{i}$ be a sequence in $R$ with 
$(O \mathcal{F}) \lim _{i} a_{i}=0$ with respect to an $(O)$-sequence $\left(\sigma_{p}\right)_{p}$. Then for every $I \in \mathcal{F}^{*}$ there exists $J \in \mathcal{F}^{*}$ such that $J \subset I$ and $(O) \lim _{i \in J} a_{i}=0$ with respect to the same $(O)$-sequence $\left(\sigma_{p}\right)_{p}$.

We now prove the main result, by means of sliding hump-type techniques.

Theorem 3.2 Let $R$ be a Dedekind complete $(\ell)$-group, $\mathcal{F}$ be a block-respecting filter of $\mathbb{N}$, $m_{n}: \Sigma \rightarrow R, n \in \mathbb{N}$, be a sequence of equibounded $\sigma$-additive measures, $\left(C_{k}\right)_{k}$ be a disjoint sequence in $\Sigma$, with

(i) (D) $\lim _{n} m_{n}\left(C_{k}\right)=0$ for any $k \in \mathbb{N}$, and

(ii) $(R D \mathcal{F}) \lim _{n} m_{n}\left(\bigcup_{k \in P} C_{k}\right)=0$ with respect to $P \in \mathcal{P}(\mathbb{N})$. Then,

3.2.1) for every strictly increasing sequence $\left(k_{n}\right)_{n}$ in $\mathbb{N}$ we get

$$
(D \mathcal{F}) \lim _{n} m_{n}\left(C_{k_{n}}\right)=0 \text {; }
$$

3.2.2) if $\mathcal{F}$ is also diagonal and $R$ is super Dedekind complete and weakly $\sigma$-distributive, then the only condition (ii) is sufficient to get (1).

Proof: For each $n \in \mathbb{N}$, set $H_{n}:=C_{k_{n}}$. Let $u:=\vee_{A \in \Sigma, n \in \mathbb{N}}\left|m_{n}(A)\right|$ : such an element does exist in $R$, thanks to equiboundedness of the $m_{n}$ 's. For each $n \in \mathbb{N}$ let $\left(a_{t, l}^{(n)}\right)_{t, l}$ be a $(D)$-sequence related with $\sigma$-additivity of $m_{n}$ and the sequence $\left(H_{n}\right)_{n}$. For every $\varphi \in \mathbb{N}^{\mathbb{N}}$ and $n \in \mathbb{N}$ there is $\bar{l} \in \mathbb{N}$, with

$$
\left|m_{n}(A)\right| \leq \bigvee_{t=1}^{\infty} a_{t, \varphi(t+n)}^{(n)} \text { for all } A \in \Sigma, A \subset \bigcup_{l=I}^{\infty} H_{l} .
$$

By the Fremlin Lemma 2.3 there is a $(D)$-sequence $\left(a_{t, l}\right)_{t, l}$ with

$$
u \wedge\left(\sum_{n=1}^{q}\left(\bigvee_{t=1}^{\infty} a_{t, \varphi(t+n)}^{(n)}\right)\right) \leq \bigvee_{t=1}^{\infty} a_{t, \varphi(t)}
$$

for each $q \in \mathbb{N}$ and $\varphi \in \mathbb{N}^{\mathbb{N}}$. From (2) and (3) it follows that for every $\varphi \in \mathbb{N}^{\mathbb{N}}$ and $n \in \mathbb{N}$ there exists $\bar{l} \in \mathbb{N}$ with $\left|m_{n}(A)\right| \leq \bigvee_{t=1}^{\infty} a_{t, \varphi(t)}$ for all $A \in \Sigma, A \subset \bigcup_{l=l}^{\infty} H_{l}$.

Let $\left(b_{t, l}\right)_{t, l}$ be a regulator, satisfying the condition of $(R D \mathcal{F})$-convergence as in (ii).

Since $(D) \lim _{n} m_{n}\left(C_{k}\right)=0$ for every $k \in \mathbb{N}$, then for each $k \in \mathbb{N}$ there exists a regulator $\left(c_{t, l}^{(k)}\right)$ such that for every $\varphi \in \mathbb{N}^{\mathbb{N}}$ there is $\bar{n} \in \mathbb{N}$ with $\left|m_{n}\left(C_{k}\right)\right| \leq \vee_{t=1}^{\infty} c_{t, \varphi(t+k)}^{(k)}$ for all $n \geq \bar{n}$. Since the $m_{n}^{t, l}$, s are equibounded, arguing analogously as above, by the Fremlin Lemma 2.3 we find a regulator $\left(c_{t, l}\right)_{t, l}$ such that for each $\varphi \in \mathbb{N}^{\mathbb{N}}$ and $k \in \mathbb{N}$ there exists $\bar{n} \in \mathbb{N}$ with $\left|m_{n}\left(C_{k}\right)\right| \leq \underset{t=1}{\infty} C_{t, \varphi(t)}$ for all $n \geq \bar{n}$.

Again by Lemma 2.3, there are two $(D)$-sequences $\left(d_{t, l}\right)_{t, l},\left(e_{t, l}\right)_{t, l}$, with

$$
\begin{gathered}
u \wedge\left(\sum _ { k = 1 } ^ { q } \left({\left.\left.\underset{t=1}{\infty} a_{t, \varphi(t+k+1)}\right)\right)}^{a_{i=1}^{\infty} d_{t, \varphi(t)},}\right.\right. \\
u \wedge\left(\sum_{k=1}^{q}\left(\bigvee_{t=1}^{\infty} C_{t, \varphi(t+k)}\right)\right) \leq \bigvee_{t=1}^{\infty} e_{t, \varphi(t)}
\end{gathered}
$$

for every $q \in \mathbb{N}$ and $\varphi \in \mathbb{N}^{\mathbb{N}}$. For every $t, l \in \mathbb{N}$, set

$$
z_{t, l}=2\left(b_{t, l}+d_{t, l}+e_{t, l}\right) .
$$

We prove that the $(D)$-sequence $\left(z_{t, l}\right)_{t, l}$ satisfies the condition of $(D \mathcal{F})$-convergence in 3.21). Otherwise there is $\varphi \in \mathbb{N}^{\mathbb{N}}$ with the property that $C:=\left\{n \in \mathbb{N}:\left|m_{n}\left(H_{n}\right)\right| \leq \vee_{t=1}^{\infty} Z_{t, \varphi(t)}\right\} \notin \mathcal{F}$.

We get that $I:=\mathbb{N} \backslash C=\left\{n \in \mathbb{N}:\left|m_{n}\left(H_{n}\right)\right| \nless \vee_{t=1}^{\infty} Z_{t, \varphi(t)}\right\} \in \mathcal{F}^{*}$ : otherwise, there would be $F \in \mathcal{F}$ with $I \cap F=\varnothing$, that is $F \subset C$ and hence $C \in \mathcal{F}$, a contradiction.

Let $N_{0}=1$. By $\sigma$-additivity of $m_{1}$, there is a cofinite subset $P_{1} \subset \mathbb{N}$, with 


$$
N_{0}<p_{1}:=\min P_{1} \text {, and } v\left(m_{1}\right)\left(F_{1}\right) \leq{\underset{t}{t=1}}^{\infty} a_{t, \varphi(t+1)},
$$

where $F_{1}:=\bigcup_{l \in P_{1}} H_{l}$. By (i) there is an integer $N_{1}>p_{1}$ with $\left|m_{n}\left(H_{l}\right)\right| \leq \bigvee_{t=1}^{\infty} C_{t, \varphi(t+1)}$ whenever $n \geq N_{1}$ and $l=1, \cdots, p_{1}$. By $\sigma$-additivity of $m_{1}, m_{2}, \cdots, m_{N_{1}}$, there is a cofinite subset $P_{2} \subset P_{1}$, with $N_{1}<p_{2}:=\min P_{2}$, and $v\left(m_{n}\right)\left(F_{2}\right) \leq \vee_{t=1}^{\infty} a_{t, \varphi(t+2)}$ for every $n=1,2, \cdots, N_{1}$, where $F_{2}:=\bigcup_{l \in P_{2}} H_{l}$. Proceeding analogously as above, we find an integer $N_{2}>p_{2}$ with $\left|m_{n}\left(H_{l}\right)\right| \leq \vee_{t=1}^{\infty} c_{t, \varphi(t+2)}$ whenever $n \geq N_{2}$ and $l=1, \cdots, p_{2}$.

By induction, it is possible to find: a strictly decreasing sequence $\left(P_{k}\right)_{k}$ of cofinite subsets of $\mathbb{N}$, a strictly decreasing sequence $\left(F_{k}\right)_{k}$ in $\Sigma$ and two strictly increasing sequences $\left(N_{k}\right)_{k},\left(p_{k}\right)_{k}$ in $\mathbb{N}$ such that, for every $k \in \mathbb{N}, N_{k}>p_{k}, p_{k+1}>N_{k} p_{k}=\min P_{k} ; F_{k}=\bigcup_{l \in P_{k}} H_{l}$;

$$
\begin{aligned}
& v\left(m_{n}\right)\left(F_{k+1}\right) \leq \bigvee_{t=1}^{\infty} a_{t, \varphi(t+k+1)}, \text { and so } v\left(m_{n}\right)\left(F_{k+1}\right) \leq{\underset{t=1}{\vee}}_{t, \varphi(t)} \text {, for any } n=1, \cdots, N_{k} ; \\
& \left|m_{n}\left(H_{l}\right)\right| \leq{\underset{t=1}{\vee} C_{t, \varphi(t+k)}}^{\infty} \text {, and so }\left|m_{n}\left(H_{l}\right)\right| \leq{\underset{t=1}{\vee} e_{t, \varphi(t)}}^{\infty} \text { for all } n \geq N_{k} \text { and } l=1, \cdots, p_{k} .
\end{aligned}
$$

Since $\mathcal{F}$ is block-respecting, there is $J:=\left\{j_{1}, j_{2}, \cdots\right\} \in \mathcal{F}^{*}, J \subset I$, with $N_{k} \leq j_{k}<N_{k+1}$ for every $k \in \mathbb{N}$. As $J \in \mathcal{F}^{*}$, then either $J_{1}:=\left\{j_{1}, j_{3}, j_{5}, \cdots\right\} \in \mathcal{F}^{*}$ or $J_{2}:=\left\{j_{2}, j_{4}, j_{6}, \cdots\right\} \in \mathcal{F}^{*}$. Without loss of generality, let $J_{1} \in \mathcal{F}^{*}$ (see also [15] [16] [18]). Put $A:=\bigcup_{h=1}^{\infty} H_{j_{2 h-1}}$. We have:

$$
\begin{aligned}
& m_{j_{1}}(A)=m_{j_{1}}\left(H_{j_{1}}\right)+m_{j_{1}}\left(H_{j_{3}} \cup H_{j_{5}} \cup \cdots\right) ; \\
& m_{j_{2 h-1}}(A)=m_{j_{2 h-1}}\left(H_{j_{1}} \cup H_{j_{3}} \cup \ldots \cup H_{j_{2 h-3}}\right)+m_{j_{2 h-1}}\left(H_{j_{2 h-1}}\right)+m_{j_{2 h-1}}\left(H_{j_{2 h-1}} \cup H_{j_{2 h+3}} \cup \cdots\right), \quad h \geq 2 .
\end{aligned}
$$

Since $j_{2 h-1}<N_{2 h-1}<p_{2 h}$ and

$$
H_{j_{2 h+1}} \cup H_{j_{2 h+3}} \cup \cdots \subset \bigcup_{l=p_{2 h+1}}^{\infty} H_{l}=F_{2 h+1} \quad \text { for every } h \in \mathbb{N},
$$

from (7) and (10) we get

$$
\left|m_{j_{2 h-1}}\left(H_{j_{2 h+1}} \cup H_{j_{2 h+3}} \cup \ldots\right)\right| \leq{\underset{t=1}{\infty}}_{t, \varphi(t)}
$$

Moreover, since $j_{2 h-3}<N_{2 h-3}<p_{2 h-2}<p_{2 h-1}$ for every $h \geq 2$, from (8) we obtain

$$
\left|m_{j_{2 h-1}}\left(H_{j_{1}} \cup H_{j_{3}} \cup \cdots \cup H_{j_{2 h-3}}\right)\right| \leq \vee_{t=1}^{\infty} e_{t, \varphi(t)} \text {. }
$$

If $\left|m_{j_{2 h-1}}(A)\right| \leq \vee_{t=1}^{\infty} b_{t, \varphi(t)}$, then from (9), (11) and (12) we have $\left|m_{j_{2 h-1}}\left(H_{j_{2 h-1}}\right)\right| \leq \bigvee_{t=1}^{\infty} Z_{t, \varphi(t)}$.

But we know that $\left|m_{j_{2 h-1}}\left(H_{j_{2 h-1}}\right)\right| \underset{t=1}{\star} \bigvee_{t, \varphi(t)}^{\infty}$, and so we get a contradiction.

Thus $\left|m_{j_{2 h-1}}(A)\right| \not \vee_{t=1}^{\infty} b_{t, \varphi(t)}$ for all $h \in \mathbb{N}$, and hence $L:=\left\{l \in \mathbb{N}:\left|m_{l}(A)\right| \not \vee_{t=1}^{\infty} b_{t, \varphi(t)}\right\} \in \mathcal{F}^{*}$.

Since, by (ii), $\mathbb{N} \backslash L \in \mathcal{F}$, we obtain that $L \cap(\mathbb{N} \backslash L) \neq \varnothing$, which is absurd. This proves 3.2.1).

3.2.2) Put $H_{n}:=C_{k_{n}}, n \in \mathbb{N}$, and let $\left(b_{t, l}\right)_{t, l}$ be a $(D)$-sequence, satisfying $(R D \mathcal{F})$-convergence in condition (ii). Of course, for every $\mathcal{F}$-stationary set $J$, the regulator $\left(b_{t, l}\right)_{t, l}$ satisfies (ii) also with respect to $(R D \mathcal{F}(J))$-convergence. Since $R$ is super Dedekind complete and weakly $\sigma$-distributive, by Theorem 2.1 there is an $(O)$-sequence $\left(\sigma_{p}\right)_{p}$, satisfying condition (ii), when $(R D \mathcal{F})$-convergence is replaced with $(R O \mathcal{F})$-or $(R O \mathcal{F}(J))$-convergence. For every $I \in \mathcal{F}^{*}$ there is $J \in \mathcal{F}^{*}, J \subset I$, with $(R O) \lim _{n \in J} m_{n}\left(C_{k}\right)=0$, $k \in \mathbb{N}$, with respect to $\left(\sigma_{p}\right)_{p}$ (see also Lemma 3.1). From this and Theorem 2.1 it follows that there is a regulator $\left(c_{t, l}\right)_{t, l}$, such that for every $I \in \mathcal{F}^{*}$ there exists $J \in \mathcal{F}^{*}, J \subset I$, with 


$$
(R D) \lim _{n \in J} m_{n}\left(C_{k}\right)=0, k \in \mathbb{N},
$$

with respect to $\left(c_{t, l}\right)_{t, l}$. Let now $\left(a_{t, l}^{(n)}\right)_{t, l}, \quad n \in \mathbb{N}$, be regulators associated to $\sigma$-additivity of the $m_{n}$ 's, $u$ be as in the proof of 3.2.1), $\left(a_{t, l}\right)_{t, l}$ be as in (3) and $\left(d_{t, l}\right)_{t, l},\left(e_{t, l}\right)_{t, l},\left(z_{t, l}\right)_{t, l}$ be as in (4), (5), (6) respec- tively. We prove that the regulator $\left(z_{t, l}\right)_{t, l}$ satisfies 3.2.2). Otherwise, by proceeding analogously as in the proof of 3.2.1), we find $I \in \mathcal{F}^{*}$ and $\varphi \in \mathbb{N}^{\mathbb{N}}$ with $\left|m_{n}\left(H_{n}\right)\right| \pm \vee_{t=1}^{\infty} Z_{t, \varphi(t)}$ for each $n \in I$. In correspondence with $I$, there is $J \in \mathcal{F}^{*}, J \subset I$, satisfying (13). Note that the sequence $m_{n}\left(H_{n}\right), n \in J$, does not $(\mathcal{F}(J))$ converge to 0 (see also [18]). Since $J \in \mathcal{F}^{*}$ and $\mathcal{F}$ is block-respecting, then, by $\operatorname{Remark} 2.4, \mathcal{F}(J)$ is block-respecting too. By 3.2.1) used with $m_{n}, n \in J$, and $\mathcal{F}(J)$, it follows that $(\mathcal{F}(J)) \lim _{n \in J} m_{n}\left(H_{n}\right)=0$, getting a contradiction. This proves 3.2.2).

A result analogous to Theorem 3.2 holds in the setting of finitely additive measures.

Theorem 3.3 Let $R$ be a Dedekind complete $(\ell)$-group, $\left(C_{k}\right)_{k}$ be as in Theorem 3.2, $\mathcal{F}$ be a blockrespecting filter of $\mathbb{N}, m_{n}: \Sigma \rightarrow R, n \in \mathbb{N}$, be an equibounded sequence of finitely additive measures, and assume that

(i) $(D) \lim _{n} m_{n}\left(C_{k}\right)=0$ for any $k \in \mathbb{N}$;

(ii) $(R D \mathcal{F}) \lim _{n} \sum_{k \in P} m_{n}\left(C_{k}\right)=0$ with respect to $P \in \mathcal{P}(\mathbb{N})$.

Then for every strictly increasing sequence $\left(k_{n}\right)_{n}$ in $\mathbb{N}$ we get

$$
(D \mathcal{F}) \lim _{n} m_{n}\left(C_{k_{n}}\right)=0 \text {. }
$$

If $\mathcal{F}$ is also diagonal and $R$ is super Dedekind complete and weakly $\sigma$-distributive, then the only condition (ii) is enough to get (14).

Indeed, it will be enough to apply Theorem 3.2 to the measures $\mu_{n}$, defined by

$$
\mu_{n}(P)=\sum_{k \in P} m_{n}\left(C_{k}\right), \quad P \subset \mathbb{N}, n \in \mathbb{N} .
$$

Analogously as Theorem 3.2 it is possible to prove a Nikodým boundedness-type theorem in the context of ( $\ell$ ) -groups and filter convergence, extending [34], Theorem 4.6 (see also [15] Lemma 3.4).

Theorem 3.4 Let $R$ be any Dedekind complete $(\ell)$-group, $u \in R, u>0, U=[-u, u], \mathcal{F}$ be a block-respecting filter of $\mathbb{N}, m_{j}: \Sigma \rightarrow R, j \in \mathbb{N}$, be a sequence of finitely additive measures, and assume that 3.4.1) for every disjoint sequence $\left(C_{n}\right)_{n}$ in $\Sigma$ and $j \in \mathbb{N}$ there is a cofinite set $Q_{j} \subset \mathbb{N}$ with $\sum_{n \in Q} m_{j}\left(C_{n}\right) \in U$ for each $Q \subset Q_{j}$.

Let $\left(C_{n}\right)_{n}$ be a disjoint sequence in $\Sigma$ and $\left(w_{n}\right)_{n}$ be an increasing sequence of positive elements of $R$. For each $n \in \mathbb{N}$, set $W_{n}:=\left[-w_{n}, w_{n}\right]$ and $V_{n}:=n W_{n}+U$. Moreover suppose that:

(i) the set $\left\{m_{n}\left(C_{p}\right): n \in \mathbb{N}\right\}$ is $(P R)$-eventually bounded by $\left(W_{n}\right)_{n}$ for each $p \in \mathbb{N}$;

(ii) the set $\left\{\sum_{p \in P} m_{j}\left(C_{p}\right): n \in \mathbb{N}\right\}$ is $(P R)-\mathcal{F}$-bounded by $\left(W_{n}\right)_{n}$ for each $P \in \mathcal{P}(\mathbb{N})$.

Then we get:

3.4.2) for every strictly increasing sequence $\left(l_{n}\right)_{n}$ in $\mathbb{N}$, the set $D:=\left\{m_{n}\left(C_{l_{n}}\right): n \in \mathbb{N}\right\}$ is $(P R)-\mathcal{F}$ bounded by $\left(V_{n}\right)_{n}$;

3.4.3) if $\mathcal{F}$ is also diagonal, then the only condition (ii) is enough in order that $D$ is $(P R)-\mathcal{F}$-bounded by $\left(V_{n}\right)_{n}$.

Proof: For every $n \in \mathbb{N}$, let $H_{n}:=C_{l_{n}}$. If the thesis of the theorem is not true, then $I:=\left\{n \in \mathbb{N}: m_{n}\left(H_{n}\right) \notin V_{n}\right\} \in \mathcal{F}^{*}$. Set $n_{0}=1$. By 3.4.1) there is a cofinite set $P_{1} \subset \mathbb{N}$, with $1<p_{1}=\min P_{1}$ and $\sum_{n \in P} m_{1}\left(H_{r}\right) \in U$ for each $P \subset P_{1}$. By (i) there is $n_{1}>p_{1}$ with $m_{j}\left(H_{l}\right) \in W_{j}$ for each $j \geq n_{1}$ and $l=1, \cdots, n_{1}$. By induction, there are a strictly decreasing sequence $\left(P_{k}\right)_{k}$ of subsets of $\mathbb{N}$ and two strictly increasing sequences $\left(n_{k}\right)_{k},\left(p_{k}\right)_{k}$ of positive integers such that, for each $k \in \mathbb{N}$,

- $n_{k}>p_{k}, \quad p_{k+1}>n_{k} ; \sum_{n \in P} m_{r}\left(H_{n}\right) \in U$ for every $r=1, \cdots, n_{k}$ and $P \subset P_{k}$;

- $m_{j}\left(H_{l}\right) \in W_{j}$ for any $j \geq n_{k}$ and $l=1, \cdots, p_{k}$.

As $\mathcal{F}$ is block-respecting, proceeding analogously as in the proof of Theorem 3.2, we find a set 
$J_{1}:=\left\{j_{1}, j_{3}, j_{5}, \cdots\right\} \in \mathcal{F}^{*}, \quad J_{1} \subset I$, with $n_{k} \leq j_{k}<n_{k+1}$ for every $k \in \mathbb{N}$. For any $h \in \mathbb{N}$ we have:

$$
\sum_{q=1}^{\infty} m_{j_{2 h-1}}\left(H_{j_{2 h+2 q-1}}\right) \in U
$$

$m_{j_{2 h-1}}\left(H_{j_{l}}\right) \in W_{2 h-1}, \quad h \geq 2, \quad l=1,3, \cdots, 2 h-3$, and

$$
m_{j_{2 h-1}}\left(H_{j_{1}} \cup H_{j_{3}} \cup \cdots \cup H_{j_{2 h-3}}\right) \in(h-1) W_{2 h-1} .
$$

Put $P^{*}:=\left\{j_{2 q-1}: q \in \mathbb{N}\right\}$. If $\sum_{p \in P^{*}} m_{j_{2 h-1}}\left(H_{p}\right) \in W_{j_{2 h-1}}$, then from (15) and (16) we get $m_{j_{2 h-1}}\left(H_{j_{2 h-1}}\right) \in h W_{2 h-1}+U \subset j_{2 h-1} W_{j_{2 h-1}}+U=V_{j_{2 h-1}}$ and $m_{j_{1}}\left(H_{j_{1}}\right) \in W_{j_{1}}+U \subset j_{1} W_{j_{1}}+U=V_{j_{1}}$. This contradicts $m_{j_{2 h-1}}\left(H_{j_{2 h-1}}\right) \notin V_{j_{2 h-1}}$. Thus $\sum_{p \in P^{*}} m_{j_{2 h-1}}\left(H_{p}\right) \notin W_{j_{2 h-1}}$ for every $h \in \mathbb{N}$, and hence $\left\{l \in \mathbb{N}: m_{l}(A) \notin W_{l}\right\} \in \mathcal{F}^{*}$. From this, arguing as in 3.2.1), we obtain a contradiction, and this proves 3.4.2). From 3.4.2), proceeding analogously as in the proof of Theorem 3.2, we get 3.4.3).

Open problems: (a) Find some versions of limit theorems with respect to some other classes of filters and/or algebras satisfying suitable properties.

(b) Find some convergence and boundedness theorems with respect to other kinds of $(s)$-boundedness, $\sigma$ additivity or boundedness.

\section{Conclusions}

The Schur, Nikodým convergence and boundedness, Vitali-Hahn-Saks and Dieudonné limits have been widely investigated in the literature since the beginning of the last century, and there are several extensions of them along different directions, concerning for example the set of definition of the involved set functions, their properties and the structure of their range. The novelties in our context are both the structure of the space in which the considered measures take values and the types of convergence: indeed we deal with filter/ideal convergence in lattice groups introduced in [1]. Moreover we use a new technique in the filter setting, inspired by those in [27]-[30] and [18], and similar to that in [20], which allows us to prove some Nikodým-type convergence and boundedness theorems with respect to filter convergence for measures defined on a $\sigma$-algebra of parts of an abstract nonempty set $G$ with a direct approach, without using earlier Schur-type results for measures defined on $\mathcal{P}(\mathbb{N})$. In this context the main properties of diagonal and block-respecting filters are used, which allow us to apply the sliding hump argument to filter convergence. So it is possible to pose the question of finding some other classes of filters for which similar theorems hold or for which they are not valid, for measures, or even not necessarily finitely additive set functions, taking values in different types of abstract structures, like topological or lattice groups or metric semigroups, and defined in algebras, which satisfy similar properties and are not necessarily $\sigma$-algebras. Note that, in general, filter convergence is not inherited by subsequences. In this context, another problem that one can pose is to find similar results on convergence and boundedness theorems for non-additive set functions, or results like some kinds of uniform $(s)$-boundedness of $\sigma$-additivity when the limits of the involved sequences are intended in the filter sense, and/or with respect some other kinds of convergence in the lattice group-context, like order convergence (see also [2]).

\section{Acknowledgements}

Our thanks to the anonymous referee for his/her remarks which improved the exposition of the paper.

\section{References}

[1] Boccuto, A., Dimitriou, X. and Papanastassiou, N. (2012) Basic Matrix Theorems for $\mathcal{T}$-Convergence in $(\ell)$-Groups. Mathematica Slovaca, 62, 885-908. http://dx.doi.org/10.2478/s12175-012-0053-6

[2] Boccuto, A. and Dimitriou, X. (2014) Convergence Theorems for Lattice Group-Valued Measures. Bentham Science Publishing, U.A.E.

[3] Boccuto, A., Dimitriou, X. and Papanastassiou, N. (2010) Convergence Theorems for the Optimal Integral in Riesz spaces. Acta Mathematica (Nitra), 13, 55-61.

[4] Boccuto, A., Dimitriou, X. and Papanastassiou, N. (2010/2011) Countably Additive Restrictions and Limit Theorems 
in ( $($ ) -Groups. Atti del Seminario Matematico e Fisico dell’ Università di Modena e Reggio Emilia, 57, 121-134. (Addendum to: “Countably Additive Restrictions and Limit Theorems in ( $\ell$ )-Groups”. Ibidem, 58, 3-10.)

[5] Boccuto, A., Dimitriou, X. and Papanastassiou, N. (2012) Limit Theorems in $(\ell)$-Groups with Respect to $(D)$-Convergence. Real Analysis Exchange, 37, 249-278.

[6] Boccuto, A., Riečan, B. and Sambucini A.R. (2009) On the Product of M-Measures in ( $\ell$ )-Groups. Australian Journal of Mathematical Analysis and Applications, 6, 1-8.

[7] Boccuto, A. and Sambucini, A.R. (1998) Abstract Integration in Convergence Groups. Atti del Seminario Matematico e Fisico dell'Università di Modena, 46, 315-333.

[8] Boccuto, A., Dimitriou, X. and Papanastassiou, N. (2010) Unconditional Convergence in Lattice Groups with Respect to Ideals. Commentationes Mathematicae, 50, 161-174.

[9] Aizpuru, A. and Nicasio-Llach, M. (2008) About the Statistical Uniform Convergence. Bulletin of the Brazilian Mathematical Society, 39, 173-182. http://dx.doi.org/10.1007/s00574-008-0078-1

[10] Aizpuru, A., Nicasio-Llach, M. and Rambla-Barreno, F. (2010) A Remark about the Orlicz-Pettis Theorem and the Statistical Convergence. Acta Mathematica Sinica, English Series, 26, 305-310. http://dx.doi.org/10.1007/s10114-010-7472-5

[11] Boccuto, A., Candeloro, D. and Sambucini, A.R. (2014) Vitali-Type Theorems for Filter Convergence Related to Vector Lattice-Valued Modulars and Applications to Stochastic Processes. Journal of Mathematical Analysis and Applications, arXiv:1401.7835.

[12] Boccuto, A. and Dimitriou, X. (2011) Some Properties of ideal $\alpha$-Convergence in $(\ell)$-Groups. International Journal of Pure and Applied Mathematics, 72, 93-99.

[13] Boccuto, A. and Dimitriou, X. (2013) Modes of Ideal Continuity of $(\ell)$-Group-Valued Measures. International Mathematical Forum, 8, 841-849.

[14] Boccuto, A., Dimitriou, X. and Papanastassiou, N. (2011) Some Versions of Limit and Dieudonné-Type Theorems with Respect to Filter Convergence for $(\ell)$-Group-Valued Measures. Central European Journal of Mathematics, 9, 1298-1311. http://dx.doi.org/10.2478/s11533-011-0083-2

[15] Boccuto, A., Dimitriou, X. and Papanastassiou, N. (2012) Uniform boundedness principle, Banach-Steinhaus and approximation theorems for filter convergence in Riesz spaces. Proceedings of International Conference on Topology and its Applications ICTA 2011, Cambridge International Science Publishing, 45-58.

[16] Boccuto, A., Dimitriou, X. and Papanastassiou, N. (2012) Schur Lemma and Limit Theorems in Lattice Groups with Respect to Filters. Mathematica Slovaca, 62, 1145-1166. http://dx.doi.org/10.2478/s12175-012-0070-5

[17] Candeloro, D. and Sambucini, A.R. (2014) Filter Convergence and Decompositions for Vector Lattice-Valued Measures. Mediterranean Journal of Mathematics, arXiv:1401.7818.

[18] Aviles Lopez, A., Cascales Salinas, B., Kadets, V. and Leonov, A. (2007) The Schur $l_{1}$ Theorem for Filters. Journal of Mathematical Physics, Analysis, Geometry, 3, 383-398.

[19] Boccuto, A., Dimitriou, X. and Papanastassiou, N. (2011) Brooks-Jewett-Type Theorems for the Pointwise Ideal Convergence of Measures with Values in $(\ell)$-Groups. Tatra Mountains Mathematical Publications, 49, 17-26. http://dx.doi.org/10.2478/v10127-011-0021-5

[20] Boccuto, A. and Dimitriou, X. (2013) Limit Theorems for Topological Group-Valued Measures. Acta Mathematica (Nitra), 16, 37-43.

[21] Boccuto, A. and Dimitriou, X. (2014) Some New Types of Filter Limit Theorems for Topological Group-Valued Measures. Real Analysis Exchange, 39, 139-174.

[22] Boccuto, A., Das, P., Dimitriou, X. and Papanastassiou, N. (2012) Ideal Exhaustiveness, Weak Convergence and Weak Compactness in Banach Spaces. Real Analysis Exchange, 37, 389-410.

[23] Boccuto, A. and Dimitriou, X. (2011) Ideal Exhaustiveness and Limit Theorems for ( $\ell$ )-Groups. Acta Matematica (Nitra), 14, 65-70.

[24] Boccuto, A. and Dimitriou, X. (2011) Some New Results on Limit Theorems with Respect to Ideal Convergence in ( $)$-Groups. Atti del Seminario Matematico e Fisico dell' Università di Modena e Reggio Emilia, 58, 163-174.

[25] Boccuto, A. and Dimitriou, X. (2013) Ideal Limit Theorems and Their Equivalence in $(\ell)$-Group Setting. Jounal of Matematics Research, 5, 43-60.

[26] Drewnowski, L. (1972) Equivalence of Brooks-Jewett, Vitali-Hahn-Saks and Nikodym theorems. Bulletin de l' Académie Polonaise des Sciences, Série des sciences mathematiques, astronomiques et physiques, 20, 725-731. 
[27] Candeloro, D. (1985) Uniforme esaustività e assoluta continuità. Bollettino dell' Unione Matematica Italiana, 4-B, 709-724.

[28] Candeloro, D. (1985) Sui teoremi di Vitali-Hahn-Saks, Dieudonné e Nikodým. Rendiconti del Circolo Matematico di Palermo Serie II, (Supplemento al), 8, 439-445.

[29] Candeloro, D. (1985) Alcuni teoremi di uniforme limitatezza. Rendiconti dell'Accademia Nazionale detta dei XL, $\mathbf{9}$, 249-260.

[30] Candeloro, D. and Letta, G. (1985) Sui teoremi di Vitali-Hahn-Saks e di Dieudonné. Rendiconti dell'Accademia Nazionale detta dei $X L, \mathbf{9}, 203-213$.

[31] Boccuto, A. (2003) Egorov Property and Weak $\sigma$-Distributivity in Riesz Spaces. Acta Mathematica (Nitra), 6, 61-66.

[32] Boccuto, A., Minotti, A.M. and Sambucini, A.R. (2013) Set-Valued Kurzweil-Henstock Integral in Riesz Space Setting. PanAmerican Mathematical Journal, 23, 57-74.

[33] Fremlin, D.H. (1975) A Direct Proof of the Matthes-Wright Integral Extension Theorem. Journal of the London Mathematical Society, s2-s11, 276-284. http://dx.doi.org/10.1112/jlms/s2-11.3.276

[34] Boccuto, A. and Candeloro, D. (2004) Uniform Boundedness Theorems in Riesz Spaces. Atti del Seminario Matematico e Fisico dell' Università di Modena e Reggio Emilia, 52, 369-382.

\section{A. M. S. Classifications}

Primary: 26E50, 28A12, 28B10, 40A35, 54A20. Secondary: 06E15, 46G12, 54H11, 54H12. 